\title{
Comunidades de aves de bosques y pastizales en los afloramientos rocosos aislados del Paraje Tres Cerros, Corrientes, Argentina
}

\author{
Blas Fandiño ${ }^{1,2}$, Juan Manuel Fernández ${ }^{3}$, María Luz Thomann ${ }^{3}$, Rodrigo Cajade ${ }^{1,3}$ \& \\ Alejandra Beatriz Hernando ${ }^{1,3}$ \\ 1. Fundación Amado Bonpland, San Juan 1182, (W3404CBN), Corrientes, Argentina. \\ 2. Ministerio de Medio Ambiente de la provincia de Santa Fe, Patricio Cullen 6161, (S3004IYC), Santa Fe, Argentina; \\ blasfand@hotmail.com \\ 3. Laboratorio de Herpetología, Departamento de Biología, Facultad de Ciencias Exactas y Naturales y Agrimensura. \\ Universidad Nacional del Nordeste. Avenida Libertad 5400, (W3404AAR), Corrientes, Argentina; \\ juanmarex@hotmail.com,mluz_26@live.com.ar, rodrigocajade@hotmail.com, alejahernando@gmail.com
}

Recibido 18-V-2016. Corregido 13-XI-2016. Aceptado 13-XII-2016.

\begin{abstract}
Bird communities of forests and grasslands on isolated rocky outcrops in Paraje Tres Cerros, Corrientes, Argentina. Isolated rocky outcrops can act as patch habitats that host great biodiversity through the diversity of microhabitats, presence of restricted taxa and the high turnover of species in the patch matrix. The isolated rocky outcrops of Paraje Tres Cerros (ARPTC), Corrientes, Argentina, are a unique landscape in the region and biologically little explored. We conducted a study to describe for the first time the bird community of the forest and grassland of the ARPTC, using 136 lists following the ten species Mackinnon method from the breeding and non-breeding seasons. We evaluated the diversity, trophic guilds and phenology of the species, and compared attributes of grassland and forest communities. The total species richness was 107, with the forest being 1.3 times more diverse than the grassland. Completeness of inventories ranged from $83-90 \%$ in the grassland and 88 to $99 \%$ in the forest. The habitat complementarity was $62 \%$, indicating the presence of several species exclusive of forest and grassland habitats. The trophic structure of communities showed a good representation of insectivorous birds in both habitats. Frugivorous birds were more frequent in the forest and granivorous birds were more frequent in the grasslands. Due to the arrival of migrant species, both habitats had significantly higher richness in the breeding season than in the non-breeding season. Our results showed similar general aspects and patterns for bird communities and with specific studies for bird communities of rocky outcrops. The present study highlights the importance of the isolated rocky outcrops of Paraje Tres Cerros for the protection of local and regional bird biodiversity and represents a benchmark for the management and the protection of these ecosystems. Rev. Biol. Trop. 65 (2): 535-550. Epub 2017 June 01.
\end{abstract}

Key words: bird assemblages, rocky ecosystems, habitat, diversity, trophic guilds, fenology.

Los afloramientos rocosos aislados se describen como paisajes destacados en biodiversidad, debido a la presencia de organismos restringidos a este hábitat y al alto recambio de especies con la matriz circundante (Gasc et al., 1998; Sarthou, Villiers, \& Ponge, 2003; Porembski, 2007; Sarthou, Kounda-kikia, Vaculika, Morab, \& Pongea, 2009). En particular, la vida silvestre animal es atraída a los afloramientos rocosos por los refugios que ofrecen las grietas y oquedades de las rocas, la presencia de bebederos naturales formados en concavidades de las rocas que retienen el agua, el microclima generado por la elevación térmica diurna de las rocas y su retención nocturna del calor y la presencia de diversas fuentes 
de alimento (Fredericksen, Fredericksen, Flores, McDonald, \& Rumiz, 2003; Michael, Lindenmayer, \& Cunningham, 2010).

A nivel global, el conocimiento generado sobre la biodiversidad de afloramientos rocosos aislados se ha concentrado principalmente en la vegetación (Sarthou et al., 2003; Porembski, 2007; Sarthou et al., 2009), mientras que la fauna de vertebrados recibió menos atención, y de la avifauna en particular, se conocen escasos antecedentes (Fredericksen et al., 2003). Los afloramientos rocosos ubicados en el Paraje Tres Cerros, Corrientes, Argentina, se caracterizan por conformar tres elevaciones aisladas entre sí que emergen sobre la llanura circundante, constituyendo verdaderos cerros (Cajade et al., 2013a; Cajade, Etchepare, Falcione, Barrasso, \& Alvarez, 2013b). Históricamente, estos afloramientos han recibido pocos aportes que brinden información de su diversidad biológica, conociéndose principalmente estudios sobre su flora (Parodi, 1943; Meregalli, 1998; Ravenna, 2003; 2009). Más recientemente y hasta la actualidad, diferentes investigaciones comenzaron a generar información sobre su fauna de vertebrados (Cajade et al., 2013a; b; Odriozola, 2015; Argoitia, 2016), incluyendo el presente estudio de avifauna.

Los estudios de diversidad de especies son apropiados cuando el objetivo del trabajo es describir las comunidades de un área relativamente poco conocida, siendo un tema central tanto en ecología de comunidades como en biología de la conservación (Bibby, 2004; Moreno, Barragán, Pineda, \& Pavón, 2011). El nordeste de Argentina y particularmente la provincia de Corrientes fueron foco de variados estudios que aportaron al conocimiento de su avifauna. Estos incluyen la revisión de colecciones (Darrieu \& Camperi, 1990), registros de especies novedosas (Chatellenaz, Fernández, \& Thomann, 2012), evaluación de poblaciones de especies amenazadas (Di Giacomo et al., 2010), pero son relativamente escasos los estudios que describen comunidades de aves (Fraga, 2001; Giraudo et al., 2003; Capllonch, Lobo, Ortiz, \& Ovejero, 2005). Estos trabajos cubrieron gran parte de los paisajes y/o ecorregiones más conspicuas de la provincia, como el Iberá (Fraga, 2001; Giraudo et al., 2003), selvas del río Uruguay (Capllonch et al., 2005) y ambientes chaqueños (Chatellenaz, 2004). Sin embargo, la avifauna de la ecorregión del Espinal, ha recibido menos atención, y particularmente los afloramientos rocosos correntinos, siendo un paisaje único y singular de esta provincia y regiones vecinas (Parodi, 1943; Meregalli, 1998; Ravenna, 2003; 2009; Cajade et al., 2013a; b) aún no ha sido estudiado.

Al considerar la importancia biológica de los afloramientos rocosos en general (Gasc et al., 1998; Sarthou et al., 2003; Fredericksen et al., 2003; Sarthou et al., 2009) y el desconocimiento de la avifauna en los afloramientos rocosos aislados del Paraje Tres Cerros en particular, el objetivo de este trabajo es describir y caracterizar las comunidades de aves de bosques y pastizales que habitan en estos ecosistemas.

\section{MATERIALES Y MÉTODOS}

Área de estudio: El estudio se realizó en el Paraje Tres Cerros, ubicado en el Departamento San Martín, en el centro este de la provincia de Corrientes, Argentina. El relieve topográfico de este paraje presenta tres afloramientos rocosos o cerros, llamados "Capará" (158 msnm, 2909'55" S - 56 51'43" W), "El Chico" (148 msnm, 2906'89" S - 56 $56^{\circ} 5^{\prime} 87^{\prime}$ " W) y "Nazareno" (179 msnm, 2906'78" S-56 55'19" W), mientras que la región más occidental de este último es referida a veces como un cuarto cerro denominado "Pelón" (131 msnm, 2906'07" S - 56 56'20" W) (Cajade et al., 2013a; b) (Fig. 1). El cerro Nazareno (83 ha) aflora a $1 \mathrm{~km}$ de distancia del Chico (34 ha), mientras que este último a $6.5 \mathrm{~km}$ del Capará (79 ha). La superficie que abarcan los tres cerros es de aproximadamente 186 ha, representados en un $60 \%$ por pastizales y un $40 \%$ por bosques. Desde el punto de vista fitogeográfico los cerros pertenecen al Distrito del Nandubay, dentro de la división biogeográfica de la Provincia del Espinal en el Dominio Chaqueño (Cabrera, 1971; Cabrera \& Willink, 1980). Geológicamente, 

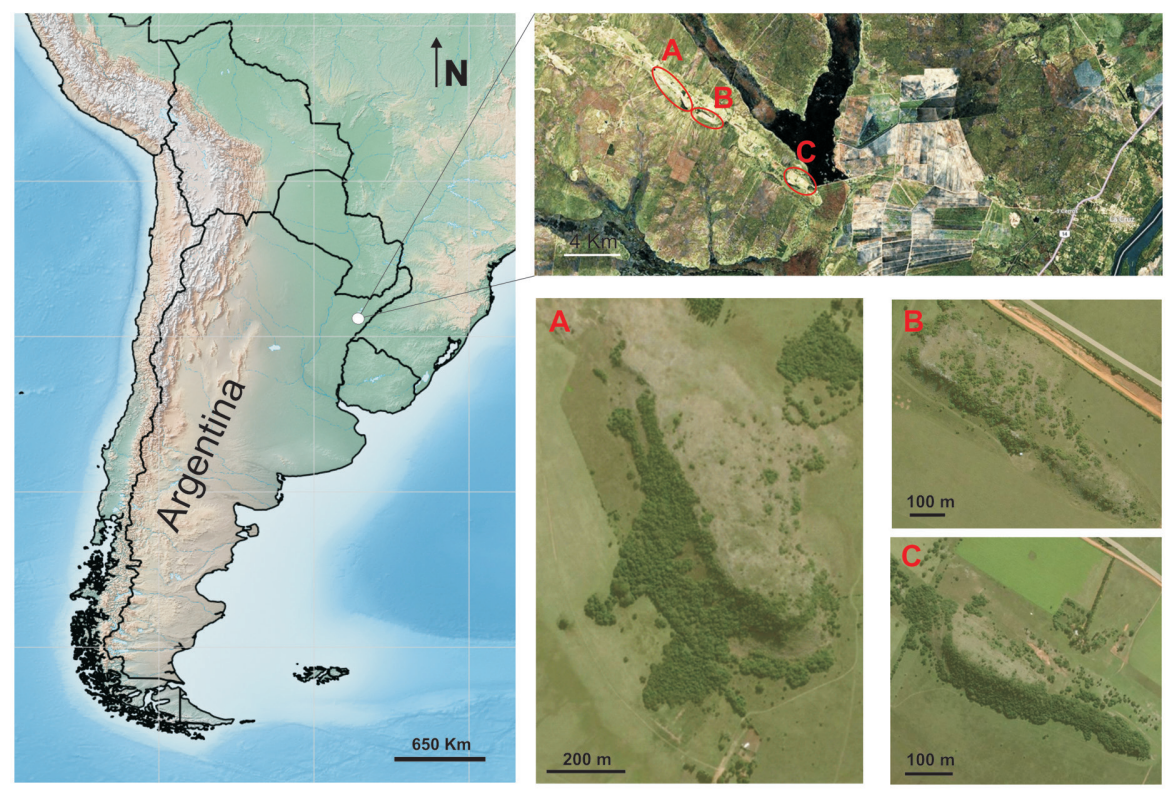

Fig. 1. Paraje Tres Cerros, Corrientes, Argentina. Los círculos ubican los cerros Nazareno (A), El Chico (B), y Capará (C).

Fig. 1. Paraje Tres Cerros. Ovals indicate the three hills: Nazareno (A), Chico (B), and Capará (C).

los afloramientos representan rocas de tipo arenisca cuarzosa pertenecientes a la formación estratigráfica Botucatú cuya edad data entre el Jurásico Superior y el Cretácico Inferior (Herbst \& Santa Cruz, 1999; Aceñolaza, 2007). Estos cerros poseen una geomorfología de dunas con cumbres truncadas que forman una divisoria topográfica de orientación SE-NW, conformando una ladera norte y una ladera sur diferenciadas en sus unidades de paisaje. La ladera norte presenta una vegetación arbustiva rala con hierbas graminosas, mientras que la ladera sur se halla cubierta por un bosque higrófilo (Parodi, 1943; Cajade et al., 2013a). En contacto con la base de los afloramientos rocosos, se halla una pendiente de erosión que finaliza en la llanura circundante (Iriondo \& Kröhling, 2008). La pendiente se caracteriza por un ambiente de pastizal con elementos florísticos típicos de serranías y por la presencia de pequeños arroyos con pequeñas lagunas artificiales de carácter permanente. La vegetación de la llanura que circunda los afloramientos y la pendiente de erosión, está representada por pastizales, pantanos y sabanas (Parodi, 1943; Cabrera, 1971).

Métodos: Se realizó el muestreo de aves de los tres afloramientos rocosos y la pendiente de erosión circundante durante 21 días distribuidos en siete períodos entre diciembre 2012 y julio 2014, cubriendo la estación reproductiva (12 días de muestreo en cuatro períodos) y no reproductiva de las aves (nueve días de muestreo en tres períodos). En cada período los tres afloramientos rocosos se muestrearon mediante listas de Mackinnon (MacKinnon \& Phillipps, 1993). Esta técnica consiste en registrar sucesivamente todas las especies vistas u oídas mientras el observador se desplaza lentamente, obteniendo consecutivamente listas de abundancias de la misma cantidad de especies (Herzog, Kessler, \& Cahill, 2002; O'Dea, Watson \& Whittaker, 2004). Es una técnica de evaluación rápida estandarizada para comunidades de aves tropicales apropiada para obtener estimaciones confiables de la riqueza de especies y para evaluar la adecuación 
del esfuerzo de muestreo realizado (Herzog et al., 2002; O'Dea et al., 2004). En este estudio se utilizó listas de diez especies, recomendado por Herzog et al. (2002) y O'Dea et al. (2004), registrando desde la primera especie detectada hasta la décima dentro del mismo hábitat (i.e. bosque o pastizal), con el objetivo de obtener datos de cada ambiente para su análisis y comparación. La acumulación de listas de Mackinnon dentro de cada hábitat, y en los siete períodos de muestreo, se realizaron mediante recorridos aleatorios en diferentes sectores de cada afloramiento rocoso. Se realizaron en total 136 listas de Mackinnon en bosques $(\mathrm{n}=112)$ y pastizales $(n=24)$ en la estación reproductiva $(\mathrm{n}=95)$ y no reproductiva $(\mathrm{n}=41)$ de laderas y pendiente de erosión de la base de los cerros, desde el amanecer y durante las cuatros horas siguientes y en las últimas horas de la tarde en respuesta a la mayor actividad de las aves (Ralph et al., 1996).

Se asignó a las especies categorías de gremios tróficos basándose en el principal componente de su dieta a partir de datos bibliográficos (Canevari, et al., 1991; Isacch, Bó, Maceira, Demaría, \& Peluc, 2003; Almeida, Couto, \& Alemida, 2003; Codesido \& Bilenca, 2004) y observaciones personales de campo. Además, se les asignó la fenología, siguiendo a MazarBarnett y Pearman (2001) y evaluación propia de registros en el área de estudio, considerando las categorías de residente, visitante estival y visitante invernal. En el ordenamiento taxonómico se siguió a Remsen et al. (2015).

\section{Análisis de datos:}

Diversidad: La riqueza de especies total y para cada hábitat se obtuvo a partir del número de especies detectadas en los muestreos sistematizados. Calculamos la diversidad verdadera en números de especies efectivas de cada hábitat con la fórmula propuesta por Jost (2006) y

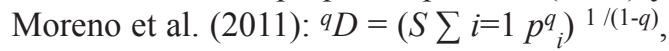
donde, $\mathrm{q} D$ es la diversidad verdadera, $p i$ es la abundancia relativa (abundancia proporcional) de la iésima especie, $S$ es el número de especies, y $q$ es el orden de la diversidad y define la sensibilidad del índice a las abundancias relativas de las especies (Jost, 2006; Moreno et al., 2011). La abundancia relativa de las especies obtenida para cada hábitat es equivalente a la fracción entre el número de individuos registrados de cada especie y el total de individuos de todas las especies.

Se evaluó la complementariedad entre hábitats mediante el índice de complementariedad (CAB) (Colwell \& Coddington, 1994; Moreno, 2001) calculada a partir de $\mathrm{Cab}=\mathrm{Uab}$ / Sab, donde Uab (número de especies únicas a cualquiera de los dos sitios) $=\mathrm{a}+\mathrm{b}-2 \mathrm{c} ; \mathrm{y}$ a es el número de especies del sitio $\mathrm{A}, \mathrm{b}$ es el número de especies del sitio $\mathrm{B}, \mathrm{y}$ c es el número de especies en común entre los sitios A y B, mientras que Sab (riqueza total para ambos sitios combinados) $=\mathrm{a}+\mathrm{b}-\mathrm{c}$. A partir de estos valores se calculó la complementariedad de los sitios A y B. Así, la complementariedad varía desde cero, cuando ambos sitios son idénticos en composición de especies, hasta uno, cuando las especies de ambos sitios son completamente distintas.

Finalmente, se evaluó la completitud de los inventarios, estimando la riqueza esperada para cada hábitat calculada con los estimadores no paramétricos Chao1, ACE (basados en la incidencia de las especies), Chao 2 y Michaelis-Menten Mean (MMMean, basados en abundancia de individuos de las especies) con el software Estimates versión 7.5 (Colwell, 2006). El porcentaje de completitud de los inventarios de los afloramientos rocosos y pendientes de erosión circundantes se obtuvo de la relación entre las especies registradas respecto al total de especies esperadas que predicen los estimadores de riqueza.

Gremios tróficos: La riqueza de especies y abundancia de individuos de cada gremio trófico se obtuvo a partir de la suma de especies e individuos registrados asignados a cada categoría. Se comparó la abundancia media de individuos de cada gremio trófico (número de individuos de cada gremio/total de listas de Mackinnon) dentro del mismo hábitat mediante un análisis de Kruskal-Wallis y post test 
de Dunn, y entre hábitats mediante la prueba no paramétrica de Wilcoxon.

Fenología: Se evaluó cómo varía la riqueza de especies entre hábitats y estaciones comparando: a) la riqueza de especies residentes y migratorias (visitante estival y visitante invernal); b) la riqueza del período reproductivo y no reproductivo entre hábitats mediante curvas de rarefacción con el software EcoSim 7.0 (Gotelli \& Entsminger, 2004). Aplicamos curvas de rarefacción debido a las diferencias halladas entre las completitudes de los inventarios de bosque y pastizal en las estaciones del año. Las curvas de rarefacción permiten comparar la riqueza de especies entre sitios en base al mismo número de individuos. Las simulaciones se corrieron 1000 veces y el número medio esperado de especies de cada hábitat y estación fue comparado basado en intervalos de confianza del $95 \%$ (la no superposición entre intervalos de confianza indica una diferencia significativa entre medias) (Blake \& Loiselle, 2001); c) cómo aportan los diferentes gremios tróficos a la variación estacional de individuos en el área considerando la residencia o migración de cada grupo mediante su abundancia media.

\section{RESULTADOS}

Diversidad: Se registró en los afloramientos rocosos y sus pendientes de erosión circundantes 107 especies incluidas en 31 familias y 15 órdenes (Anexo 1). Los bosques son habitados por 29 especies más que los pastizales (80 vs. 51) (Cuadro 1), complementándose estos hábitats en un $62 \%$. Del total de especies registradas, 56 (52\%) ocurrieron sólo en bosques y $28(26 \%)$ sólo en pastizales, mientras que $23(21 \%)$ en ambos hábitats. El número de especies efectivas evidencia que el bosque $\left({ }^{1} D=35.27\right)$ es 1.3 veces más diverso que el pastizal $\left({ }^{1} D=27.11\right)$.

La completitud del inventario es elevada tal como indican los estimadores para ambos ambientes (Cuadro 1). Un inventario más completo se obtuvo en los bosques (estimación de 88 a $99 \%$ ) y un menor porcentaje de completitud en los pastizales (83 a $90 \%$ ). Todos los estimadores predicen una mayor riqueza en los bosques, donde son esperables de una a once nuevas especies, mientras que en los pastizales de seis a diez nuevas especies (Cuadro 1).

Gremios tróficos: Se registraron los siguientes 10 gremios tróficos: Carnívoro (C),

CUADRO 1

Datos de las muestras tomadas y valores de riqueza estimada en bosques y pastizales de los afloramientos rocosos aislados del Paraje Tres Cerros, Corrientes, Argentina

TABLE 1

Data samples and values of estimated richness for forests and grasslands at the isolated rocky outcrops Paraje Tres Cerros, Corrientes, Argentina

\begin{tabular}{lcccccccc}
\multicolumn{1}{r}{ Hábitat } & \multicolumn{1}{c}{ Datos } & \multicolumn{5}{c}{ Riqueza estimada } \\
& $\mathrm{N}$ & Ind. & Sobs & ACE & Chao 1 & Chao 2 & MMMean \\
Pastizal & 24 & 507 & 51 & 57 & 59 & 58 & 61 \\
Bosque & 112 & 1773 & 80 & 86 & 86 & 91 & 81 \\
\hline
\end{tabular}

$\mathrm{N}=$ Número de muestras. Ind = Individuos detectados. Sobs = Especies registradas. Chao1, ACE, Chao 2 y MichaelisMenten Mean $=$ estimadores no paramétricos.

$\mathrm{N}=$ number of samples. Ind $=$ Individuals detected. About $=$ Species registered. Chao1, ACE Chao 2 and Michaelis-Menten Mean $=$ nonparametric estimators. 

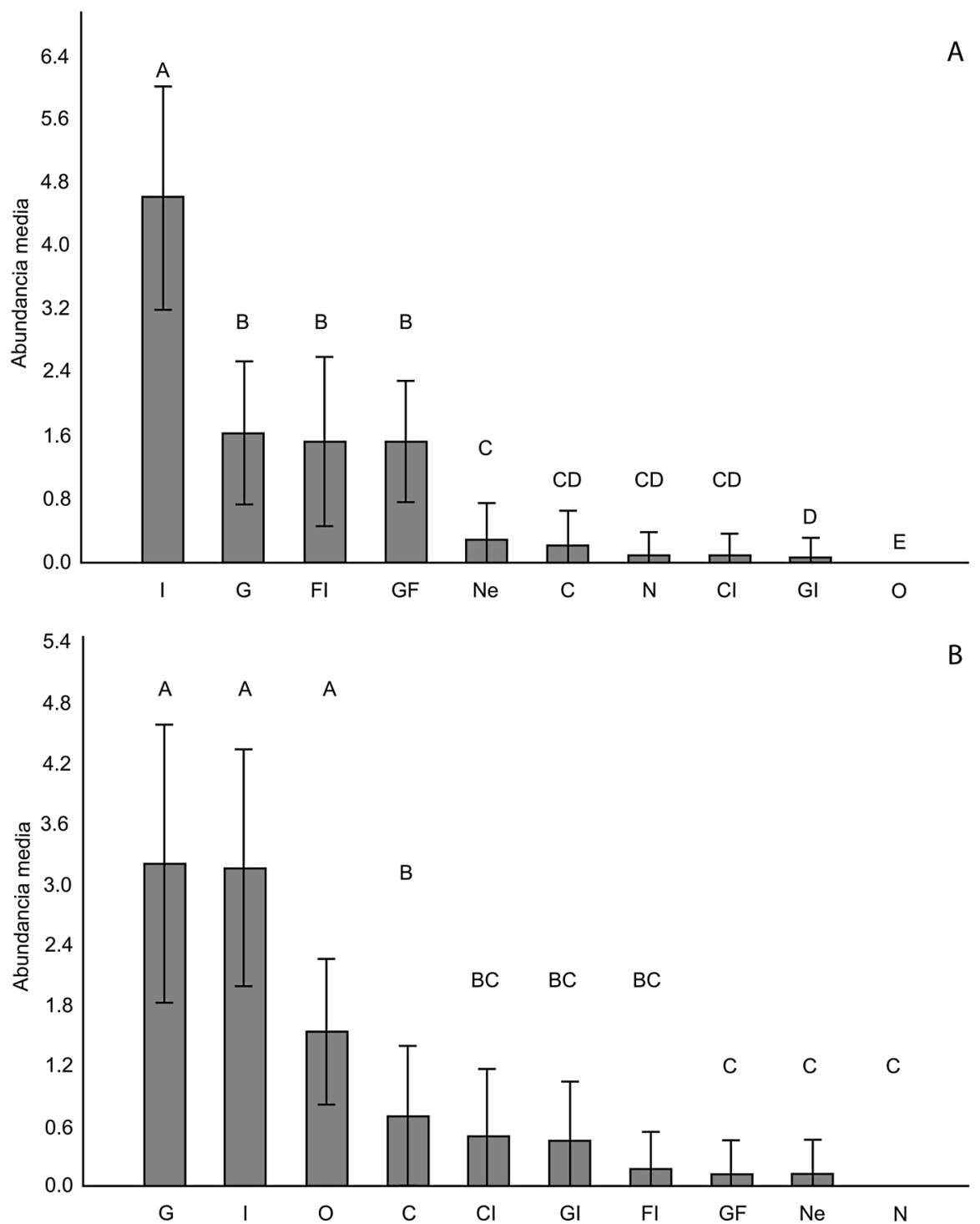

Fig. 2. Comparación en bosques (Fig. 2A) y pastizales (Fig. 2B) de la abundancia media de individuos (barras \pm desviación estándar) de gremios tróficos representados en los afloramientos rocosos del Paraje Tres Cerros, Corrientes, Argentina: Carnívoro (C), Carnívoro-Insectívoro (CI), Frugívoro-Insectívoro (FI), Granívoro (G), Granívoro-Frugívoro (GF), Granívoro-Insectívoro (GI), Insectívoro (I), Necrófago (Ne), Nectarívoro (N) y Omnívoro (O). Prueba no paramétrica de Kruskal-Wallis, bosque: $\mathrm{H}=676.82, \mathrm{p}<0.0001$; pastizal: $\mathrm{H}=136.97, \mathrm{p}<0.0001$; letras diferentes asignadas a cada barra en el interior del gráfico indican diferencias significativas de abundancias de individuos entre gremios tróficos.

Fig. 2. Comparison of average abundance of individuals (bars \pm standard deviation) of trophic guilds between forest (A) and grasslands (B) of the rocky ourtcrops of Paraje Tres Cerros, Corrientes, Argentina. References: Carnivore (C), Carnivore-Insectivore (CI), Frugivore-Insectivore (FI), Granivore (G), Granivore-Frugivore (GF), Granivore-Insectivore (GI), Insectivore (I), Necrophage (Ne), Nectarivore (N) and Omnivore (O ). Nonparametric Kruskal-Wallis test, forest: $\mathrm{H}=676.82, \mathrm{p}<0.0001$; pasture: $\mathrm{H}=136.97, \mathrm{p}<0.0001$; different letters assigned to each bar within the graph indicate significant differences in abundances of individuals between trophic guilds. 
Carnívoro-Insectívoro (CI), Frugívoro-Insectívoro (FI), Granívoro (G), Granívoro-Frugívoro (GF), Granívoro-Insectívoro (GI), Insectívoro (I), Necrófago (Ne), Nectarívoro (N) y Omnívoro (O, más de dos ítems). En los afloramientos rocosos los gremios tróficos mejor representados fueron el I ( $\mathrm{s}=49)$, seguido del $\mathrm{G}(\mathrm{s}=17)$ y del F-I $(\mathrm{s}=11)$. Particularmente en los bosques, el gremio I fue el más rico $(\mathrm{s}=40)$ y con mayor abundancia difiriendo significativamente de los restantes gremios en número de individuos (Fig. 2A). En orden decreciente de riqueza específica le siguen los gremios F-I ( $(\mathrm{s}=11), \mathrm{G}(\mathrm{s}=9)$ y G-F $(\mathrm{s}=6)$, difiriendo significativamente en abundancia de los otros gremios (Fig. 2A). En los pastizales, también el $\mathrm{I}(\mathrm{s}=20)$ fue el más diverso, seguido del $\mathrm{G}(\mathrm{s}=$ 14) y el C ( $\mathrm{s}=5)$. Sin embargo, el $\mathrm{G}$ presentó la mayor abundancia de individuos, que seguido del I y O, difirieron significativamente del resto de los gremios tróficos (Fig. 2B). Las especies de aves que consumen frutas y otro ítem fueron más abundantes en bosques (i.e. F-I y G-F), mientras que gremios $\mathrm{O}$ y $\mathrm{G}$, o $\mathrm{C}$ junto a otro ítem, fueron más abundantes en pastizales (i.e. C, C-I, G, y G-I). Tres gremios no presentaron diferencias de abundancias entre hábitats, de los cuales los I y $\mathrm{Ne}$ ocurrieron en bosques y pastizales y el $\mathrm{N}$ sólo en bosques (Cuadro 2).

Fenología: El $80 \%$ de las especies residen todo el año y el restante $20 \%$ son migrantes. Entre las migrantes, el $18 \%$ ocurren en el período reproductivo (Visitantes estivales) y el $2 \%$ en el no reproductivo (Visitantes invernales). Los bosques presentan una mayor riqueza y proporción de especies migratorias que los pastizales (18 especies vs $7 ; 21 \%$ vs $12 \%$; respectivamente), incluyendo la ocurrencia exclusiva de las 2 especies migrantes

CUADRO 2

Comparación de la abundancia media de individuos de los gremios tróficos entre bosques y pastizales en los afloramientos rocosos del Paraje Tres Cerros, Corrientes, Argentina

TABLE 2

Comparison of average abundance of individuals of the trophic guilds between forests and grasslands at rocky outcrops of Paraje Tres Cerros, Corrientes, Argentina

\begin{tabular}{ccccccccccc} 
GT & \multicolumn{3}{c}{ AM } & \multicolumn{3}{c}{ R } & & VI & & VE \\
C & $0.29 \pm 0.68$ & $0.75 \pm 0.79$ & $*$ & 0.29 & 0.75 & - & - & - & - \\
CI & $0.16 \pm 0.64$ & $1.50 \pm 3.05$ & $*$ & 0.16 & 1.50 & - & - & - & - \\
FI & $2.29 \pm 1.89$ & $0.21 \pm 0.51$ & $*$ & 1.87 & 0.21 & - & - & 0.43 & - \\
G & $3.04 \pm 1.94$ & $6.13 \pm 3.60$ & $*$ & 2.98 & 6.00 & - & - & 0.05 & 0.13 \\
GF & $2.61 \pm 1.71$ & $0.21 \pm 0.59$ & $*$ & 2.61 & 0.21 & - & - & - & - \\
GI & $0.09 \pm 0.37$ & $3.71 \pm 6.37$ & $*$ & 0.09 & 3.71 & - & - & - & - \\
I & $6.19 \pm 2.77$ & $5.75 \pm 3.84$ & NS & 4.91 & 4.54 & 0.08 & - & 1.20 & 1.21 \\
N & $0.09 \pm 0.29$ & - & NS & 0.09 & & - & - & - & - \\
Ne & $1.07 \pm 2.28$ & $0.25 \pm 0.68$ & NS & 1.07 & 0.25 & - & - & - & - \\
O & - & $1.61 \pm 0.66$ & $*$ & - & 1.61 & - & - & - & - \\
\hline
\end{tabular}

Abundancia media en el ciclo anual (AM) ( \pm desvío estándar) y abundancia disgregada entre aves residentes $(\mathrm{R})$, visitantes invernales (VI) y visitantes estivales (VE) para bosques (B) y pastizales (P) por gremios tróficos (GT) (ver siglas en metodología). Prueba no paramétrica de Wilcoxon comparando la abundancia media de individuos de cada gremio trófico entre bosques y pastizales en el ciclo anual; $*=\mathrm{P}<0.001 ; \mathrm{NS}=$ no significativo.

Mean abundance in the annual cycle (AM) ( \pm standard deviation) and splitted abundance among resident birds $(\mathrm{R})$, winter visitors (VI) and summer visitors (VE) for forests (B) and grasslands (P) by trophic guilds (GT) (see acronym methodology). Nonparametric Wilcoxon test comparing the average abundance of individuals of each trophic guild between forests and grasslands in the annual cycle; $*=\mathrm{P}<0.001 ; \mathrm{NS}=$ not significant. 
invernales (Anexo 1). Una mayor riqueza de especies residentes también ocurre en los bosques, pero la proporción de este grupo es mayor en los pastizales (61 especies vs $45 ; 76$ vs 88 $\%$; respectivamente) (Anexo 1). Así, la mayor riqueza de especies se da en la estación reproductiva, con 78 especies registradas en los bosques y 51 en los pastizales, mientras que en la estación no reproductiva, la riqueza disminuye en ambos ambientes perdiendo un $19 \%$ en bosques y $12 \%$ en pastizales, alcanzando las 63 y 45 especies, respectivamente. Las curvas de rarefacción reflejan estas diferencias de riqueza entre los bosques en la estación reproductiva respecto a los bosques en la estación no reproductiva, y pastizales durante el ciclo anual. Los pastizales en la estación reproductiva son más ricos en especies que los bosques y pastizales en la estación no reproductiva, y ambos ambientes no difieren en riqueza en la estación no reproductiva (Fig. 3).

Sólo especies de tres grupos tróficos son migratorias (i.e. I, FI, G), mientras que los restantes siete gremios se componen sólo de especies residentes. Los I migrantes estivales ocurren con abundancias similares en bosques y pastizales, y los visitantes invernales sólo en bosques. Los visitantes estivales FI ocurren sólo en los bosques, mientras que los $\mathrm{G}$ en ambos ambientes pero con mayor abundancia en los pastizales (Cuadro 2).

\section{DISCUSIÓN}

El número de especies registradas en este estudio representa un $21 \%$ de las aproximadamente 500 especies de aves mencionadas en las 8.8 millones de ha de la provincia de Corrientes (Capllonch et al., 2005; Chatellenaz, Cano, Saibene, \& Ball, 2010; De la Peña, 2012). Este porcentaje es significativo considerando las 186 ha que abarcó el área de estudio, y su potencialidad para el incremento de la riqueza de especies, tal como indican los estimadores calculados. Así, el Paraje

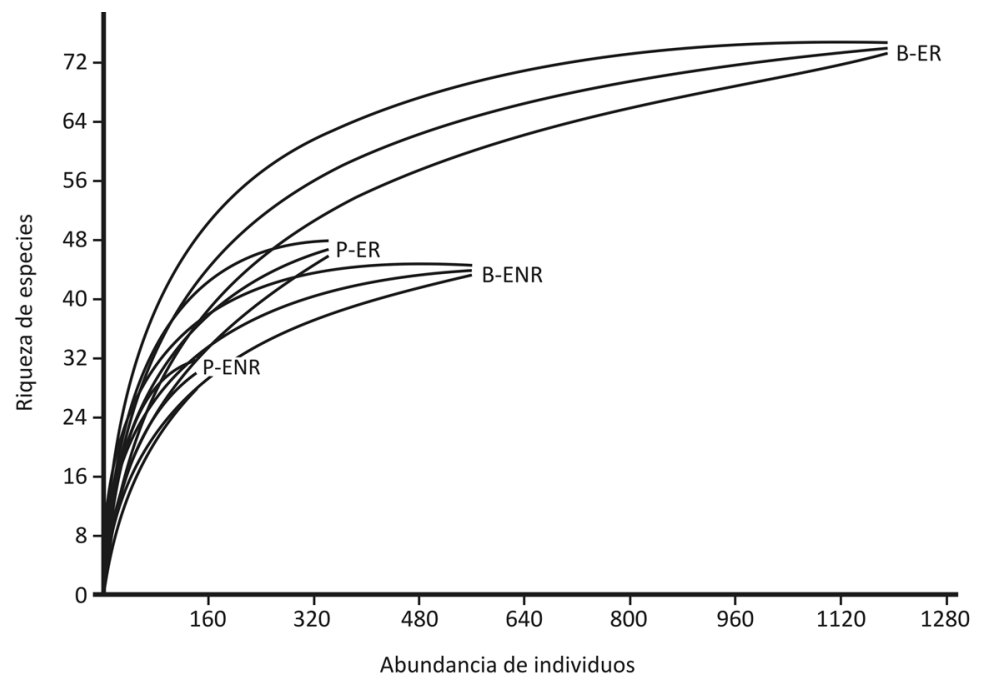

Fig. 3. Curvas de rarefacción basada en la abundancia de individuos con sus intervalos de confianza del $95 \%$, comparando el número de especies entre bosques $(\mathrm{B})$ y pastizales $(\mathrm{P})$ y entre la estación reproductiva (ER) y no reproductiva (ENR) en los afloramientos rocosos del Paraje Tres Cerros, Corrientes, Argentina. Referencias: B-ER = bosque en la estación reproductiva; $\mathrm{B}-\mathrm{ENR}=$ bosque en la estación no reproductiva; $\mathrm{P}-\mathrm{ER}=$ pastizal en la estación reproductiva; $\mathrm{P}-\mathrm{ENR}=$ pastizal en la estación no reproductiva.

Fig. 3. Rarefaction curves based on the abundance of individuals (confidence intervals of $95 \%$ ), comparing the number of species between forest (B) and grasslands (P) and between breeding season (ER) and non-breeding season (ENR) at rocky outcrops Paraje Tres Cerros, Corrientes, Argentina. References: B-ER = forest in the breeding season; B-ENR = forest in the non-breeding season; P-ER = pasture in the breeding season; P-ENR = pasture in the non-breeding season. 
Tres Cerros constituye un lugar de importancia para la diversidad de avifauna, principalmente para las aves de bosque, debido a la escasa representación de este hábitat en una región naturalmente dominada por una matriz de pastizales. La mayor diversidad registrada en los bosques respecto a los pastizales es coincidente con lo hallado en otras regiones del noreste de Argentina como Iberá (provincia de Corrientes) (Giraudo \& Ordano, 2003), el valle de inundación del río Paraná (provincias de Chaco y Santa Fé) (Chatellenaz, 2005; Fandiño, 2011) y la región chaqueña (provincias de Chaco y Santa Fé) (Chatellenaz et al., 2010; Fandiño, 2011). Este patrón es explicado por la mayor diversidad de especies vegetales y la complejidad fisonómica y estructural de los bosques, que ofrecen un mayor número de nichos $\mathrm{y}$ variada disponibilidad de recursos para la nidificación, alimentación, refugio, perchar, entre otros (MacArthur \& MacArthur, 1961; McIntyre, 1995; Villard, Trzcinski, \& Merriam, 1999). La elevada complementariedad entre bosques y pastizales señala que ambos ambientes poseen especies propias que responden a requerimientos particulares relacionados a los diferentes recursos que ofrece cada uno de éstos hábitats (Halffter \& Moreno, 2005).

Los gremios tróficos insectívoro, nectarívoro y necrófago no presentaron diferencias de abundancia de individuos entre bosques y pastizales. La alta representatividad de insectívoros indica disponibilidad y abundancia de recursos para este grupo en los afloramientos rocosos estudiados. Entre todos los grupos funcionales de aves, el insectívoro es el más rico en especies (Sekercioglu, 2006) por lo que suele ser el más abundante respecto a los otros gremios (Codesido \& Bilenca, 2004; Fandiño, Berduc, \& Beltzer, 2010; Zurita \& Bellocq, 2012). Sin embargo, podría considerarse la influencia propia de los ecosistemas rocosos para albergar una mayor riqueza de aves insectívoras. En concordancia con nuestros resultados se ha documentado una mayor riqueza y abundancia de aves insectívoras en afloramientos rocosos aislados de Bolivia, muy similares a los aquí descritos (Fredericksen et al., 2003). Al respecto, se hipotetiza que la capacidad de las rocas de absorber calor rápidamente y luego liberarlo gradualmente al caer el sol, crea un microclima favorable respecto de la matriz circundante que atrae numerosos insectos que son presas de estas aves (Fredericksen et al., 2003). El gremio nectarívoro estuvo escasamente representado y sólo en bosques, postulándose que este grupo tiende a evitar las áreas abiertas (Tscharntke et al., 2008; Zurita \& Bellocq, 2012). Las aves necrófagas fueron relativamente abundantes en ambos ambientes, lo que puede ser explicado por el uso observado de estos hábitats para diferentes funciones, como nidificar y perchar en bosques y volar sobre los pastizales buscando alimento. Además, esta abundancia estaría relacionada con otro recurso que ofrecen las rocas (en pastizales rocosos y en bosques de la ladera de los cerros) como es la liberación de calor y la generación de corrientes térmicas aprovechada por las aves planeadoras de las familias Cathartidae (e.g. Cathartes aura y Coragyps atratus) Accipitridae (e.g. Buteogallus meridionalis) y Falconidae (e.g. Caracara plancus) (Fredericksen et al., 2003).

Otros gremios tróficos presentan diferencias de abundancia entre ambientes. La mayor abundancia de aves frugívoras (i.e. frugívorasinsectívoras y granívoras-frugívoras) en los bosques ha sido también descrita en otras regiones del noreste de Argentina (Zurita \& Bellocq, 2012) y estaría relacionada a la producción de frutos ornitócoros de este hábitat. En este estudio observamos especies frugívoras-insectívoras (e.g. Elaenia parvirostris, Empidonomus varius y Turdus spp.) y granívoras-frugívoras (e.g. Saltator similis y Euphonia chlorotica) removiendo frutos de diversas especies vegetales del bosque (e.g. Guarea macrophylla, Lythraea molleoides, Ficus sp., Phytolacca dioica, Allophylus edulis, Phoradendrom sp., Myrsine laetevirens, Eugenia uniflora y Smilax campestris). Además, las especies frugívoras aquí registradas son típicas de bosques que nidifican en árboles $u$ otras formas vegetales (e.g. arbustos, lianas o enredaderas) en ambientes boscosos (De la Peña, 2013; obs. pers.), 
lo que explica también su mayor abundancia. Los gremios tróficos más abundantes (i.e. granívoros e insectívoros) o exclusivos (i.e omnívoros) de pastizales responden a la fisonomía y disponibilidad de recursos de este ambiente. Por ejemplo, las especies omnívoras registradas (i.e. Rhea americana, Rhynchotus rufescens y Nothura maculosa), son especialistas de estos ambientes donde forrajean y nidifican (Setubal, 1991; Bellis, Martella, Navarro, \& Vignolo, 2004), mientras que la abundancia de aves granívoras estaría relacionada a que a este tipo de dieta está claramente asociado a ambientes dominados por gramíneas (Isacch et al., 2003; Zurita \& Bellocq, 2012), donde además varios paseriformes registrados de este gremio nidifican (i.e. Donacospiza albifrons, Sicalis luteola, Emberizoides herbicola, Emberizoides ypiranganus, Embernagra platensis, Volatinia jacarina, Sporophila caerulescens, Ammodramus humeralis, Sporagra magellanica) (De la Peña, 2013; obs. pers.).

Los resultados muestran que la estructura del ensamble de aves varía estacionalmente, con una mayor riqueza en la estación reproductiva en ambos hábitats debido al arribo de las especies migrantes, patrón concordante con lo ya descrito para bosques (Cueto \& Lopez de Casenave, 2000; Codesido \& Bilenca, 2004) y pastizales (Isacch \& Martínez, 2001; Isacch et al., 2003) del sur de Sudamérica. El Litoral argentino, que incluye el área de estudio, es considerado una de las vías migratorias más importantes de Argentina, donde la mayoría de las especies migrantes estivales que arriban del cono sur se reproducen (Capllonch, Ortiz, \& Soria, 2008; de la Peña, 2013). El mayor incremento de riqueza y abundancia en la estación reproductiva de frugívoros insectívoros en bosques e insectívoros en bosques y pastizales responde principalmente al arribo de tiránidos, familia de dieta principalmente insectívora y gremio trófico con mayores variaciones estacionales (Karr, 1976).

Los afloramientos rocosos aislados del Paraje Tres Cerros han permanecido biológicamente inexplorados por mucho tiempo. Nuestros resultados demuestran la importancia de estos ecosistemas para albergar una significativa proporción de las aves de la región, incluyendo especies cercanas a la amenaza a escala global y amenazadas a escala nacional (i.e. Rhea americana, Polystictus pectoralis y Emberizoides ypiranganus) (AA/AOP \& SAyDS, 2008; BirdLife International, 2016). La escasa superficie que abarcan los afloramientos rocosos estudiados (186 ha) y la singular riqueza de su flora y fauna (Cajade et al., 2013a; b, este estudio) resaltan la necesidad de conservar estos ecosistemas. Recientemente, la protección de la naturaleza de los cerros Nazareno y Chico fue garantizada por la creación de la "Reserva Natural Privada Paraje Tres Cerros" (RNPPTC) (107 ha), pero será necesario realizar esfuerzos en gestiones para incluir el cerro Capará bajo alguna categoría de protección. El conocimiento generado sobre la estructura y comunidad de aves contribuirá con la conservación de la naturaleza de los bosques y pastizales de estos afloramientos rocosos, tanto como herramienta a utilizarse en planes de manejo de la RNPPTC como para contribuir en la gestión para lograr la protección del cerro Capará. La presencia de especies amenazadas registradas en los afloramientos y alrededores (e.g. Buteogallus coronatus, Alectrurus risora, Sporophila palustris, Gubernatrix cristata) (Fandiño, 2012; Cajade et al., 2013a; obs. pers.) sugiere la oportunidad de gestionar la creación de un AICA (Área de Importancia para la Conservación de Aves; Di Giacomo, 2005) que abarque el Paraje Tres Cerros. Este reconocimiento otorgado por Bird Life International y su contra parte local Aves Argentinas, podrá sumar esfuerzos de gestión para la protección y conservación de la naturaleza del Paraje Tres Cerros y sus afloramientos rocosos.

\section{AGRADECIMIENTOS}

A los propietarios y trabajadores de las Estancias La Higuera Cué, Las Marías y Buena Vista por recibirnos amablemente en sus establecimientos en el Paraje Tres Cerros. A la Dirección de Recursos Naturales, Ministerio de Turismo de la Provincia de Corrientes 
por los permisos de trabajo brindados. A Conservation, Research and Education Opportunities International (CREOI), Secretaria General de Ciencia y Técnica de la Universidad Nacional del Nordeste (UNNE) y Comisión Nacional de Investigaciones Científicas y Técnicas (CONICET) por el financiamiento brindado. A Walter Medina y José Miguel Piñeiro por su colaboración en el campo. A Martjan Lammertink por la revisión del Abstract. A los revisores anónimos quienes mejoraron el manuscrito con sus comentarios.

\section{RESUMEN}

Los afloramientos rocosos aislados se describen como paisajes destacados en biodiversidad debido a la gran variedad de microhabitats, presencia de taxones restringidos y al alto recambio de especies con la matriz circundante. Particularmente los afloramientos rocosos aislados del Paraje Tres Cerros (ARPTC), Corrientes, Argentina, representan un paisaje único en la región, biológicamente fueron poco explorados. Se estudió la comunidad de aves de bosques y pastizales de los ARPTC mediante 136 listas de Mackinnon de diez especies durante la temporada reproductiva y no reproductiva. Se evaluó la diversidad, riqueza estimada y completitud de inventarios, abundancia de gremios tróficos y fenología de las especies. Se comparó estos atributos entre los bosques y pastizales. Se registraron 107 especies donde el bosque es 1.3 veces más diverso que el pastizal. La completitud de inventarios varió de 83 a $90 \%$ en el pastizal y de 88 a $99 \%$ en el bosque. Los hábitats se complementaron en un $62 \%$ indicando una elevada ocurrencia de especies exclusivas. Las aves insectívoras fueron abundantes en ambos ambientes, mientras que las frugívoras en bosques y las granívoras en pastizales. Ambos hábitats son significativamente más ricos en especies en la estación reproductiva por la llegada de los migrantes estivales. Los resultados concuerdan con los patrones generales conocidos para las comunidades de aves y con otros específicos para las comunidades de aves de afloramientos rocosos. El presente estudio resalta la importancia de los afloramientos rocosos aislados de Paraje Tres Cerros para la protección de la biodiversidad de aves a nivel local y regional y representa una herramienta de manejo para la conservación de estos ecosistemas.

Palabras clave: ensambles de aves, ecosistemas rocosos, hábitat, diversidad, gremios tróficos, fenología.

\section{REFERENCIAS}

AA/AOP \& SAyDS. (2008). Categorización de las aves de la Argentina según su estado de conservación (Informe de Aves Argentinas). Buenos Aires, Argentina: AOP, Secretaría de Ambiente y Desarrollo Sustentable.

Aceñolaza, F. G. (2007). Geología y recursos geológicos de la Mesopotamia Argentina. Tucumán, Argentina: Serie Correlación Geológica 22, INSUGEO.

Almeida, A., Couto, H., \& Almeida, A. (2003). Diversidade beta de aves em hábitats secundários da Pré-Amazônia maranhense e interação com modelos nulos. Ararajuba, 11, 157-171.

Argoitia, M. A. (2016). Inventario y conservación de la quiropterofauna del Paraje Tres Cerros, Corrientes, Argentina. (Trabajo Final de Graduación). Universidad Nacional del Nordeste, Corrientes, Argentina.

Bellis, L. M., Martella, M. B., Navarro, J. L., \& Vignolo, P. E. (2004). Home range of greater and lesser rheas in Argentina: relevance to conservation. Biodiversity and Conservation, 13, 2589-2598.

Bibby, C. J. (2004). Bird diversity survey methods. In W. J. Sutherland, I. Newton, \& R. Green, (Eds.), Bird Ecology and Conservation: A Handbook of Techniques (pp. 1-15). Oxford: Oxford University Press.

BirdLife International. (2016). IUCN Red List for birds. United Kingdom: IUCN. Retrieved from http://www. birdlife.org on 16/03/2016

Blake, J. G., \& Loiselle, B. A. (2001). Birds assemblages in second-growth and old-growth forest, Costa Rica: perspectives from mist nest and point counts. Auk, $118,304-326$.

Cabrera, A. L. (1971). Fitogeografía de la República Argentina. Boletín de la Sociedad Argentina de Botánica, XIV, 1-50.

Cabrera, A. L., \& Willink, A. (1980). Biogeografía de América Latina. Washington DC: Secretaría General de la Organización de los Estados Americanos.

Cajade, R., Medina, W., Salas, R., Fandiño, B., Paracampo, A., ... Álvarez B. B. (2013a). Las islas rocosas del Paraje Tres Cerros: un refugio de biodiversidad en el litoral mesopotámico argentino. Biológica, 16, 147-159.

Cajade, R., Etchepare, E. G., Falcione, C., Barrasso, D. A., \& Alvarez, B. B. (2013b). A new species of Homonota (Reptilia: Squamata: Gekkota: Phyllodactylidae) endemic to the hills of Paraje Tres Cerros, Corrientes Province, Argentina. Zootaxa, 3709, 162-176.

Canevari, M., Canevari, P., Carrizo, G. R., Harris, G., Rodriguez-Mata, J., \& Straneck, R. J. (1991). Nueva guia de las aves argentinas. Tomo II. Buenos Aires, Argentina: Fundación Acindar.

Capllonch, P., Lobo, R., Ortiz, D., \& Ovejero, R. (2005). La avifauna de la selva de galería en el noreste de Corrientes, Argentina: Biodiversidad, Patrones 
de Distribución y Migración. INSUGEO, Miscelánea, 14, 483-498.

Capllonch, P., Ortiz, D., \& Soria, K. (2008). Importancia del litoral fluvial argentino como corredor migratorio de aves. INSUGEO, Miscelánea, 17, 107-120.

Chatellenaz, M. L. (2004). La avifauna del bosque de Quebracho Colorado y Urunday del noroeste de Corrientes, Argentina. Facena, 20, 3-12.

Chatellenaz, M. L. (2005). Aves del valle del Río Paraná en la Provincia del Chaco, Argentina: Riqueza, Historia Natural y Conservación. INSUGEO, Miscelánea, $14,527-550$.

Chatellenaz, M. L., Fernández, J. M., \& Thomann, M. L. (2012). Nuevos registros de Macropsalis forcipata, Cyanocorax caeruleus y Pyroderus scutatus (Aves: Caprimulgidae, Corvidae y Cotingidae) en la provincia de Corrientes, Argentina. Facena, 28, 69-73.

Chatellenaz, M. L., Cano, P. D., Saibene, C., \& Ball, H. A. (2010). Inventario de las aves del Parque Nacional Mburucuyá (Provincia de Corrientes, Argentina). Acta Zoológica Lilloana, 54, 139-16.

Codesido, M. A., \& Bilenca, D. (2004). Variación estacional de un ensamble de aves en un bosque subtropical semiárido del chaco argentino. Biotropica, $36,544-554$

Colwell, R. K., \& Coddington, J. A. (1994). Estimating terrestrial biodiversity through extrapolation. Philosophical Transactions of the Royal Society, Series B345, 101-118.

Colwell, R. K. (2006). EstimateS: Statistical estimation of species richness and shared species from samples. Version 7.5. Retrieved from http://purl.oclc.org/ estimates

Cueto, V. R., \& Lopez de Casenave, J. (2000). Bird assemblages of protected and exploited coastal woodlands in east-central Argentina. Wilson Bulletin, $112,395-402$.

Darrieu, A. C., \& Camperi, A. R. (1990). Estudio de una colección de aves de Corrientes. I. (Dendrocolaptidae - Furnariidae). El Hornero, 13, 138-146.

De la Peña, M. R. (2012). Citas, observaciones y distribución de aves argentinas: Edición ampliada. Santa Fe, Argentina: Ediciones Biológica $\mathrm{N}^{\circ}$.

De La Peña, M. R. (2013). Nidos y reproducción de las aves argentinas. Santa Fe, Argentina: Ediciones Biológica $\mathrm{N}^{\circ} 8$.

Di Giacomo, A. S. (2005). Áreas importantes para la conservación de las aves en la Argentina. Sitios prioritarios para la conservación de la biodiversidad. Buenos Aires, Argentina: Aves Argentinas/Asociación Ornitológica del Plata.
Di Giacomo, A. S., Vickery, P. D., Casañas, H., Spitznagel, O. A., Ostrosky, C., Krapovickas, S., \& Bosso, A. J. (2010). Landscape associations of globally threatened grassland birds in the Aguapey river Important Bird Area, Corrientes, Argentina. Bird Conservation International, 20, 62-73.

Fandiño, B., Berduc, A. J., \& Beltzer, A. H. (2010). Ensambles de aves de bosques nativos y exóticos en la estación reproductiva de un área protegida en el espinal de Entre Ríos, Argentina. Ornitologia Neotropical, 21, 1-16.

Fandiño, B. (2011). Patrones de diversidad de aves en Santa Fe y efectividad de las áreas protegidas en su conservación. (Tesis de Doctorado). Universidad Nacional del Litoral, Santa Fe, Argentina.

Fandiño, B. (2012). Avifauna de la Estancia Loma Alta (Corrientes, Argentina). (Informe Técnico). Buenos Aires, Argentina: Fundación Hábitat y Desarrollo.

Fraga, R. M., (2001). The avifauna of Estancia San Juan Poriahú, Iberá Marshes, Argentina: Checklist and some natural history notes. Cotinga, 16, 81-86.

Fredericksen, N. J., Fredericksen, T. S., Flores, B., McDonald, E., \& Rumiz, D. (2003). Importance of granitic rock outcrops to vertebrate species in a Bolivian tropical forest. Tropical Ecology, 44, 185-196.

Gasc, J. P., Sarthou, C., Garrouste, R., Villiers, J. F., Cremers, G., \& Thiollay, J. M. (1998). Inselbergsetsavanes-rochesenGuyane: biodiversite' et conservation des milieuxassocie's aux affleurementsgranitiques. Jatba, 40, 311-327.

Giraudo, A., \& Ordano, M. (2003). Patrones de diversidad de las aves del Ibera: Variación de la riqueza, abundancia y diversidad entre localidades, regiones y hábitat. In B. B. Álvarez (Ed.), Fauna del Ibera (pp. 235-256). Corrientes, Argentina: Universidad Nacional del Nordeste.

Giraudo, A. R, Chatellenaz, M. L., Saibene, C. A., Ordano, M. A., Krauczuk, E. R., Alonso, J., \& Di Giacomo, A. S. (2003). Avifauna del Iberá: Composición y datos sobre su historia natural. In B. B. Álvarez (Ed.), Fauna del Iberá (pp. 195-223). Corrientes, Argentina: Universidad Nacional del Nordeste.

Gotelli, N. J., \& Entsminger, G. L. (2004). EcoSim: Null models software for ecology. Version 7. Acquired Intelligence Inc. \& Kesey-Bear. Jericho, VT 05465. Rtrieved from http://garyentsminger.com/ecosim/ index.htm

Halffter, G., \& Moreno, C. E. (2005). Significado biológico de las diversidades alfa, beta y gamma. En G. Halffter, J. Soberón, P. Koleff, \& A. Melic (Eds.), Sobre diversidad biológica: el significado de las diversidades alfa, beta y gamma (pp. 5-18). Zaragoza, España: Sociedad Entomológica Aragonesa. 
Herbst, R. \& Santa Cruz, J. N. (1999). Mapa litoestragráfia de la provincia de Corrientes. D'Orbiniana, 2, 1-69.

Herzog, S. K., Kessler, M., \& Cahill, T. M. (2002). Estimating species richness of tropical bird communities from rapid assessment data. The Auk, 119, 749-769.

Iriondo, M. H. \& Kröhling, D. (2008). Cambios ambientales en la cuenca del Uruguay (desde el presente hasta dos millones de años atrás). Santa Fe, Argentina: CCT, Ediciones UNL.

Isacch, J. P., \& Martínez, M. M. (2001). Estacionalidad y relaciones con la estructura del hábitat de la comunidad de aves de pastizales de Paja Colorada (Paspalum quadrifarium) manejados con fuego en la provincia de Buenos Aires, Argentina. Ornitología Neotropical, 12, 345-354.

Isacch, J. P., Bó, M. S., Maceira, N. O., Demaría, M. R., \& Peluc, S. (2003). Composition and seasonal changes of the bird community of natural grasslands in the western pampa of Argentina. Journal of Field Ornithology, 54, 59-65.

Jost, L. (2006). Entropy and diversity. Oikos, 113, 363-375.

Karr, J. R. (1976). Seasonality, resource availability, and community diversity in tropical bird communities. The American Naturalist, 110, 973-995.

MacArthur, R. H., \& MacArthur, J. W. (1961). On Bird Species Diversity. Ecology, 42, 594-598.

Mackinnon, S., \& Phillipps, K. (1993). A Field Guide to the Birds of Borneo, Sumatra, Java and Bali. Oxford: Oxford University Press.

Michael, D. R., Lindenmayer, D. B., \& Cunningham, R. B. (2010). Managing rock outcrops to improve biodiversity conservation in Australian agricultural landscapes. Ecological Management and Restoration, 11, 43-50.

Mazar-Barnett, J., \& Pearman, M. (2001). Lista comentada de las aves argentinas / Annotated checklist of Argentina. Barcelona, España: Lynx Edicions.

McIntyre, N. (1995). Effects of forest patch size on avian diversity. Landscape Ecology, 10, 85-99.

Meregalli, M., (1998). Gymnocalycium angelae spec. nov., eine neue Art aus Argentinien. Kakteen Sukk, 49, 283-290.

Moreno, C. E., (2001). Métodos para medir la biodiversidad. Zaragoza, España: M\&T-Manuales y Tesis SEA

Moreno, C. E., Barragán, F., Pineda, E., \& Pavón, N. P. (2011). Reanalizando la diversidad alfa: alternativas para interpretar y comparar información sobre comunidades ecológicas. Revista Mexicana de Biodiversidad, 82, 1249-1261.
O'dea, N., Watson, J. E. M., \& Whittaker, R. J. (2004). Rapid assessment in conservation research: a critique of avifaunal assessment techniques illustrated by Ecuadorian and Madagascan case study data. Diversity and Distributions, 10, 55-63.

Odriozola, M. (2015). Complementariedad y solapamiento entre los nichos espacial y temporal de cuatro especies sintópicas de lagartijas del Paraje Tres Cerros, Corrientes, Argentina" (Trabajo Final de Graduación). Universidad Nacional del Nordeste, Corrientes, Argentina.

Parodi, L. R. (1943). La vegetación del departamento San Martín en Corrientes, Argentina. Darwiniana, $6,127-178$

Porembski, S. (2007). Tropical inselbergs: habitat types, adaptive strategies and diversity patterns. Revista Brasileira Botanica, 30, 579-586.

Ralph, C. J., Geupel, G. R., Pyle, P., Martin, T. E., DeSante, D. F, \& Milá, B. (1996). Manual de métodos de campo para el monitoreo de aves terrestres (General Technical Report PSW-GTR-159). Albany, CA, USA: Pacific Southwest Research Station, Forest Service, U.S. Department of Agriculture.

Ravenna, P., (2003). Decisive proof on the validity of Amaryllis over Hippeastrum as mainly a South American genus, including new species and new records of Amaryllidaceae from Argentina, Barazil, and Paraguay. Onira Botanical Leaflets, 9, 9-22.

Ravenna, P., (2009). A survey in the genus Cypella and itsallies (Iridaceae). Onira Botanical Leaflets, 12, 1-10.

Remsen, J. V., Cadena, C. D., Jaramillo, A., Nores, M., Pacheco, J. F., Pérez-Emán, J., ... Zimmer, K. J. (2015). A classification of the bird species of South America. Chicago, IL, USA: American Ornithologists' Union. Retrieved from http://www.museum.lsu. edu/ Remsen/SACCBaseline.html

Sarthou, C., Villiers, J. F., \& Ponge, J. F. (2003). Shrub vegetation on tropical granitic inselbergs in French Guiana. Journal of Vegetation Science, 14, 645-652.

Sarthou, C., Kounda-Kikia, C., Vaculika, A., Morab, P., \& Pongea, J. F. (2009). Successional patterns on tropical inselbergs: A case study on the Nouragues inselberg (French Guiana). Flora, 204, 396-407.

Sekercioglu, C. H. (2006). Ecological significance of bird populations. Handbook of the Birds of the World, $11,15-51$.

Setubal, S. S. (1991). Biologia e ecologia dos tinamídeos Rhynchotus rufescens (Temminck, 1815) e Nothura maculosa (Temminck, 1855) na região do Distrito Federal, Brasil. (Tésis de Maestría). Pontifícia Universidade Católica do Rio Grande do Sul, Porto Alegre, Brasil. 
Tscharntke, T., Sekercioglu, C. H., Dietsch, T. V., Sodhi, N. S., Hoehn, P., \& J. Tylianakis (2008). Landscape constraints on functional diversity of birds and insects in tropical agroecosystems. Ecology, 89, 944-951.

Villard, M., Trzcinski, M. K., \& Merriam, G. (1999). Fragmentation effects on forest birds: Relative influence of woodland cover and configuration on landscape occupancy. Conservation Biology, 13, 774-783.

Zurita, G. A, \& Bellocq, M. I. (2012). Bird assemblages in anthropogenic habitats: identifying a suitability gradient for native species in the Atlantic Forest. Biotropica, 44, 412-419. 


\section{ANEXO 1}

Especies de aves de los afloramientos rocosos aislados del Paraje Tres Cerros, Corrientes, Argentina, detallando su abundancia relativa en bosques y pastizales, fenología y gremios tróficos

\section{APPENDIX 1}

Bird species from the isolated rocky outcrops of Paraje Tres Cerros, Corrientes, Argentina, detailing its relative abundance in forests and grasslands, phenology and trophic guilds

\begin{tabular}{|c|c|c|c|c|}
\hline FAMILIA/Especies & Bos & Pas & $\mathrm{Fe}$ & GT \\
\hline \multicolumn{5}{|l|}{ RHEIDAE } \\
\hline Rhea americana & & 3.35 & $\mathrm{R}$ & $\mathrm{O}$ \\
\hline \multicolumn{5}{|l|}{ TINAMIDAE } \\
\hline Rhynchotus rufescens & & 3.16 & $\mathrm{R}$ & $\mathrm{O}$ \\
\hline Nothura maculosa & & 5.92 & $\mathrm{R}$ & $\mathrm{O}$ \\
\hline \multicolumn{5}{|l|}{ ARDEIDAE } \\
\hline Syrigma sibilatrix & 0.06 & 0.79 & $\mathrm{R}$ & $\mathrm{CI}$ \\
\hline \multicolumn{5}{|l|}{ THRESKIORNITHIDAE } \\
\hline Theristicus caerulescens & 0.11 & & $\mathrm{R}$ & $\mathrm{CI}$ \\
\hline \multicolumn{5}{|l|}{ CATHARTIDAE } \\
\hline Cathartes aura & 6.49 & 1.18 & $\mathrm{R}$ & $\mathrm{Ne}$ \\
\hline Coragyps atratus & 0.28 & & $\mathrm{R}$ & $\mathrm{Ne}$ \\
\hline \multicolumn{5}{|l|}{ ACCIPITRIDAE } \\
\hline Circus cinereus & & 0.39 & $\mathrm{R} ?$ & $\mathrm{C}$ \\
\hline Circus buffoni & & 0.59 & $\mathrm{R}$ & $\mathrm{C}$ \\
\hline Buteogallus meridionalis & 0.06 & & $\mathrm{R}$ & $\mathrm{C}$ \\
\hline Rupornis magnirostris & 0.96 & & $\mathrm{R}$ & $\mathrm{C}$ \\
\hline \multicolumn{5}{|l|}{ CHARADRIIDAE } \\
\hline Vanellus chilensis & & 4.93 & $\mathrm{R}$ & I \\
\hline \multicolumn{5}{|l|}{ COLUMBIDAE } \\
\hline Patagioenas picazuro & 7.50 & 1.18 & $\mathrm{R}$ & G \\
\hline Patagioenas maculosa & 0.23 & & $\mathrm{R}$ & G \\
\hline Leptotila verreauxi & 9.81 & 0.20 & $\mathrm{R}$ & GF \\
\hline Leptotila rufaxilla & 1.52 & & $\mathrm{R}$ & GF \\
\hline Columbina picui & 0.39 & 0.20 & $\mathrm{R}$ & G \\
\hline Zenaida auriculata & 2.31 & 1.18 & $\mathrm{R}$ & G \\
\hline \multicolumn{5}{|l|}{ CUCULIDAE } \\
\hline Piaya cayana & 0.39 & & $\mathrm{R}$ & I \\
\hline Coccyzus melacoryphus & 0.23 & & VE & I \\
\hline Crotophaga ani & 0.11 & & $\mathrm{R}$ & I \\
\hline Guira guira & 0.79 & 5.92 & $\mathrm{R}$ & $\mathrm{CI}$ \\
\hline Tapera naevia & 0.34 & & VE & I \\
\hline \multicolumn{5}{|l|}{ STRIGIDAE } \\
\hline Megascops choliba & 0.06 & & $\mathrm{R}$ & $\mathrm{CI}$ \\
\hline Athene cunicularia & & 0.39 & $\mathrm{R}$ & $\mathrm{CI}$ \\
\hline \multicolumn{5}{|l|}{ CAPRIMULGIDAE } \\
\hline Setopagis parvula & 0.06 & & $\mathrm{R}$ & I \\
\hline Hydropsalis torquata & 0.28 & & $\mathrm{R}$ & I \\
\hline \multicolumn{5}{|l|}{ TROCHILIDAE } \\
\hline Chlorostilbon lucidus & 0.06 & & $\mathrm{R}$ & $\mathrm{N}$ \\
\hline
\end{tabular}

\begin{tabular}{|c|c|c|c|c|}
\hline FAMILIA/Especies & Bos & Pas & $\mathrm{Fe}$ & GT \\
\hline Hylocharis chrysura & 0.51 & & $\mathrm{R}$ & $\mathrm{N}$ \\
\hline \multicolumn{5}{|l|}{ PICIDAE } \\
\hline Melanerpes candidus & 0.06 & & $\mathrm{R}$ & $\mathrm{I}$ \\
\hline Colaptes melanochloros & 0.85 & 0.20 & $\mathrm{R}$ & $\mathrm{I}$ \\
\hline Colaptes campestris & & 1.58 & $\mathrm{R}$ & $\mathrm{I}$ \\
\hline \multicolumn{5}{|l|}{ FALCONIDAE } \\
\hline Caracara plancus & 0.79 & 0.79 & $\mathrm{R}$ & $\mathrm{C}$ \\
\hline Milvago chimango & & 1.58 & $\mathrm{R}$ & $\mathrm{C}$ \\
\hline Falco sparverius & 0.06 & & $\mathrm{R}$ & $\mathrm{C}$ \\
\hline Falco femoralis & & 0.20 & $\mathrm{R}$ & $\mathrm{C}$ \\
\hline \multicolumn{5}{|l|}{ PSITTACIDAE } \\
\hline Myiopsitta monachus & 1.41 & 0.79 & $\mathrm{R}$ & GF \\
\hline \multicolumn{5}{|l|}{ FURNARIIDAE } \\
\hline Furnarius rufus & 1.18 & 0.59 & $\mathrm{R}$ & $\mathrm{I}$ \\
\hline Phacellodomus ruber & 0.62 & & $\mathrm{R}$ & I \\
\hline Anumbius annumbi & & 0.99 & $\mathrm{R}$ & I \\
\hline Synallaxis spixi & 0.11 & & $\mathrm{R} ?$ & I \\
\hline Synallaxis frontalis & 3.78 & & $\mathrm{R}$ & $\mathrm{I}$ \\
\hline Schoeniophylax phryganophilus & 0.11 & 4.54 & $\mathrm{R}$ & I \\
\hline \multicolumn{5}{|l|}{ TYRANNIDAE } \\
\hline Elaenia spectabilis & 0.17 & & VE & FI \\
\hline Elaenia parvirostris & 1.86 & & VE & FI \\
\hline Camptostoma obsoletum & 1.30 & & $\mathrm{R}$ & $\mathrm{I}$ \\
\hline Serpophaga subcristata & 1.47 & 0.20 & $\mathrm{R}$ & I \\
\hline Polystictus pectoralis & & 0.79 & VE & I \\
\hline Euscarthmus meloryphus & 0.06 & & VE & I \\
\hline Hemitriccus margaritaceiventer & 0.39 & & $\mathrm{R}$ & I \\
\hline Myiophobus fasciatus & 0.23 & & VE & I \\
\hline Lathrotriccus euleri & 1.80 & & VE & I \\
\hline Cnemotriccus fuscatus & 0.06 & & $\mathrm{R}$ & I \\
\hline Pyrocephalus rubinus & & 0.39 & $\mathrm{R}$ & $\mathrm{I}$ \\
\hline Knipolegus cyanirostris & 0.45 & & VI & I \\
\hline Xolmis irupero & & 0.20 & $\mathrm{R}$ & I \\
\hline Machetornis rixosa & 0.23 & 1.18 & $\mathrm{R}$ & I \\
\hline Pitangus sulphuratus & 4.51 & 0.99 & $\mathrm{R}$ & I \\
\hline Myiodynastes maculatus & 0.56 & & $\mathrm{VE}$ & I \\
\hline Megarynchus pitangua & 0.68 & & $\mathrm{R}$ & I \\
\hline Empidonomus varius & 0.06 & & $\mathrm{VE}$ & FI \\
\hline Tyrannus melancholicus & 0.51 & 0.59 & $\mathrm{VE}$ & $\mathrm{I}$ \\
\hline Tyrannus savana & 1.69 & 3.75 & VE & $\mathrm{I}$ \\
\hline
\end{tabular}




\begin{tabular}{|c|c|c|c|c|c|c|c|c|c|}
\hline FAMILIA/Especies & Bos & Pas & $\mathrm{Fe}$ & GT & FAMILIA/Especies & Bos & Pas & $\mathrm{Fe}$ & GT \\
\hline Myiarchus swainsoni & 0.68 & & $\mathrm{VE}$ & I & Poospiza melanoleuca & 0.17 & & $\mathrm{R}$ & G \\
\hline Myiarchus ferox & 0.23 & & $\mathrm{VE}$ & I & Sicalis luteola & & 5.13 & $\mathrm{R}$ & G \\
\hline Myiarchus tyrannulus & 0.11 & & $\mathrm{R}$ & I & Emberizoides herbicola & & 2.37 & $\mathrm{R}$ & G \\
\hline TITYRIDAE & & & & & Emberizoides ypiranganus & & 0.20 & $\mathrm{R}$ & G \\
\hline Pachyramphus viridis & 0.11 & & $\mathrm{R}$ & FI & Embernagra platensis & & 3.16 & $\mathrm{R}$ & $\mathrm{G}$ \\
\hline Pachyramphus polychopterus & 0.62 & & $\mathrm{VE}$ & FI & Volatinia jacarina & & 0.20 & VE & G \\
\hline VIREONIDAE & & & & & Sporophila caerulescens & 0.34 & 0.39 & VE & G \\
\hline Cyclarhis gujanensis & 0.90 & & $\mathrm{R}$ & I & Coryphospingus cucullatus & 0.96 & & $\mathrm{R}$ & $\mathrm{G}$ \\
\hline Vireo olivaceus & 0.96 & & $\mathrm{VE}$ & I & INCERTAE SEDIS & & & & \\
\hline HIRUNDINIDAE & & & & & Saltator coerulescens & 0.11 & & $\mathrm{R}$ & GF \\
\hline Progne tapera & 0.28 & 0.59 & $\mathrm{VE}$ & I & Saltator similis & 3.44 & & $\mathrm{R}$ & GF \\
\hline Tachycineta leucorrhoa & & 0.20 & $\mathrm{R}$ & I & EMBERIZIDAE & & & & \\
\hline TROGLODYTIDAE & & & & & Ammodramus humeralis & & 3.35 & $\mathrm{R}$ & G \\
\hline Troglodytes aedon & 4.85 & 1.58 & $\mathrm{R}$ & I & Zonotrichia capensis & 7.05 & 7.10 & $\mathrm{R}$ & $\mathrm{G}$ \\
\hline POLIOPTILIDAE & & & & & CARDINALIDAE & & & & \\
\hline Polioptila dumicola & 2.14 & & $\mathrm{R}$ & I & Piranga flava & 0.23 & & $\mathrm{R}$ & FI \\
\hline TURDIDAE & & & & & PARULIDAE & & & & \\
\hline Turdus leucomelas & 1.02 & & $\mathrm{R}$ & FI & Setophaga pitiayumi & 1.13 & & $\mathrm{R}$ & I \\
\hline Turdus rufiventris & 0.73 & & $\mathrm{R}$ & FI & Myiothlypis leucoblephara & 0.51 & & $\mathrm{R}$ & $\mathrm{I}$ \\
\hline Turdus amaurochalinus & 4.00 & 0.99 & $\mathrm{R}$ & FI & Basileuterus culicivorus & 5.08 & & $\mathrm{R}$ & I \\
\hline MIMIDAE & & & & & ICTERIDAE & & & & \\
\hline Mimus saturninus & 0.11 & 1.18 & $\mathrm{R}$ & I & Icterus pyrrhopterus & 0.39 & & $\mathrm{R}$ & FI \\
\hline Mimus triurus & 0.06 & & VI & I & Pseudoleistes virescens & & 16.96 & $\mathrm{R}$ & GI \\
\hline MOTACILLIDAE & & & & & Agelaioides badius & 0.11 & & $\mathrm{R}$ & GI \\
\hline Anthus lutescens & & 0.20 & $\mathrm{R}$ & I & Molothrus bonariensis & 0.45 & & $\mathrm{R}$ & GI \\
\hline Anthus hellmayri & & 2.56 & $\mathrm{R}$ & I & Sturnella superciliaris & & 0.59 & $\mathrm{R}$ & GI \\
\hline THRAUPIDAE & & & & & FRINGILLIDAE & & & & \\
\hline Paroaria coronata & 0.23 & 0.79 & $\mathrm{R}$ & G & Spinus magellanica & & 0.39 & $\mathrm{R}$ & G \\
\hline Thraupis sayaca & 5.30 & & $\mathrm{R}$ & FI & Euphonia chlorotica & 0.17 & & $\mathrm{R}$ & GF \\
\hline
\end{tabular}

Bos = bosques; Pas = pastizales. $\mathrm{Fe}=$ Fenología $; \mathrm{R}=$ Residentes; $\mathrm{VE}=$ Visitante estival (Migrantes Australes del Norte); $\mathrm{VI}=$ Visitante invernal (Migrantes Australes del Sur). GT = Gremios tróficos; $\mathrm{C}=$ Carnívoro; CI = Carnívoro-Insectívoro; FI= Frugívoro-Insectívoro; $\mathrm{G}$ = Granívoro; GF = Granívoro-Frugívoro; GI = Granívoro-Insectívoro; I = Insectívoro; Ne = Necrófago; $\mathrm{N}=$ Nectarívoro; $\mathrm{O}=$ Omnívoro. La nomenclatura de las especies sigue a Remsen et al. (2015).

Bos $=$ forest; Pas = grasslands. $\mathrm{Fe}=$ Phenology; $\mathrm{R}=$ Residents; VE = Visitor summer (austral migrants Northern); VI = winter visitor (Migrants Southern South). GT = trophic Guilds; $\mathrm{C}=$ Carnivorous; $\mathrm{CI}=$ Carnivorous-Insectivorous; $\mathrm{FI}$ = frugivorous-Insectivorous; $\mathrm{G}$ = granivorous; $\mathrm{GF}$ = granivorous-frugivorous; $\mathrm{GI}$ = granivorous-Insectivorous; $\mathrm{I}=$ Insectivorous; $\mathrm{Ne}=$ Ghoul; $\mathrm{N}$ = nectarivorous; $\mathrm{O}=$ Omnivorous. The species nomenclature follows Remsen et al. (2015). 